\title{
A REMARK ON COMMUTABLE FUNCTIONS AND CONTINUOUS ITERATIONS
}

\section{MAREK KUCZMA}

The purpose of this note is to apply some recent results of $\mathrm{B}$. Choczewski to a question concerning commutable functions and continuous iterations. Namely, we shall prove the following:

TheOREM. Let $f(x)$ be a function of class $C^{2}$ in an interval $(a, b\rangle$, such that $f(x)>x$ in $(a, b), f(b)=b, \lim _{x \rightarrow a+0} f(x)=a, f^{\prime}(x)>0$ in $(a, b\rangle$, and $f^{\prime}(b)<1 .{ }^{1}$ Moreover let us assume that the function $\left(d^{2} / d x^{2}\right) f^{-1}(x)$ satisfies a Lipschitz condition in a left neighbourhood of the point $b\left(f^{-1}(x)\right.$ denotes the inverse function of $f(x)$, which exists since $\left.f^{\prime}(x)>0\right)$.

Then there exists a unique one-parameter family of functions $\phi_{s}(x)$, $s \in(-\infty, \infty)$, satisfying the following conditions for all $s$ :

(A) $\phi_{s}(x)$ is of class $C^{2}$ in $(a, b)$.

(B) $\phi_{s}(x) \in(a, b\rangle$ for $x \in(a, b\rangle, \phi_{s}(b)=b$.

(C) $\phi_{s}(x)$ commutes with $f(x)$, i.e.

$$
f\left[\phi_{s}(x)\right]=\phi_{s}[f(x)] .
$$

The family of the functions $\phi_{s}(x)$ is also, in a sense, the "best" family of continuous iterations of the function $f(x)$. The functions $\phi_{s}(x)$ may be obtained as the limit of the sequence defined by

$$
\phi_{s, n}(x)=f^{-n}\left(\phi_{s, 0}\left[f^{n}(x)\right]\right), x \in(a, b\rangle, \quad n=1,2,3, \cdots,
$$

where $f^{k}(x)$ denotes the kth iterate of the function $f(x)$, i.e., $f^{0}(x)=x$, $f^{k+1}(x)=f\left[f^{k}(x)\right], \quad f^{k-1}(x)=f^{-1}\left[f^{k}(x)\right], \quad k=0, \quad \pm 1, \pm 2, \cdots$, and $\phi_{s, 0}(x)$ is an arbitrary function of class $C^{2}$ in $(a, b\rangle$ such that

$$
\phi_{s, 0}(x) \in(a, b\rangle \text { for } x \in(a, b\rangle, \phi_{s, 0}(b)=b, \phi_{s, 0}^{\prime}(b)=\left[f^{\prime}(b)\right]^{s} .
$$

Furthermore, if the function $f(x)$ is of class $C^{r}(r>2)$ in $(a, b\rangle$, then the functions $\phi_{s}(x)$ are also of class $C^{r}$ in $(a, b)$.

Proof. In order to simplify the notation we write

$$
h(x) \stackrel{d f}{=} f^{-1}(x) .
$$

Received by the editors October 30, 1961.

${ }^{1}$ The number $a$ may also equal $-\infty$; but $b$ must be finite, which undoubtedly is a defect of the present theorem. The condition $\lim _{x \rightarrow a+0} f(x)=a$ may be omitted, but then the functions $\phi_{s}(x)$ occurring in the thesis of the theorem may not be defined in the whole interval $(a, b\rangle$, but only in a left neighbourhood of the point $b$. The conditions $f(x)>x$ in $(a, b), f^{\prime}(b)<1$, may be replaced by $f(x)<x$ in $(a, b), f^{\prime}(b)>1$. And naturally, the endpoints, $a$ and $b$, of the interval in question may be interchanged. 
Then equation (1) takes the form

$$
\phi(x)=h(\phi[f(x)]) .
$$

Moreover, we have

$$
h^{\prime}(b)\left[f^{\prime}(b)\right]^{2}=f^{\prime}(b)<1 .
$$

Consequently, as a direct consequence of a theorem of B. Choczewski [2], it follows that for every number $s \in(-\infty, \infty)$ there exists exactly one function $\phi_{s}(x)$ satisfying equation (4), and fulfilling conditions (A), (B), and the condition ${ }^{2}$

$$
\phi_{s}^{\prime}(b)=\left[f^{\prime}(b)\right]^{s} .
$$

(Condition (C) is fulfilled, since the functions $\phi_{s}(x)$ satisfy equation (4), which is equivalent to (1)).

It follows from the uniqueness of $\phi_{s}$ that $\phi_{k}(x)=f^{k}(x)$ for integral $k$. In particular we have $\phi_{1}(x)=f(x)$.

The paper by B. Choczewski has not yet been published; and since the method employed by him is essential to our further considerations, we shall present here a very short outline of his arguments, naturally omitting all details. ${ }^{3}$

Let $R$ be the space of all functions $\phi(x)$ of class $C^{2}$ in the interval $\langle b-\eta, b\rangle$ (where $\eta$ is a sufficiently small, fixed positive number, depending only on $f(x)$ and $s$ ), fulfilling the conditions:

$$
\phi(x) \in\langle a+\alpha, b\rangle \text { for } x \in\langle b-\eta, b\rangle, \phi(b)=b, \quad \phi^{\prime}(b)=\left[f^{\prime}(b)\right]
$$

$$
\left|\phi^{\prime \prime}(x)\right| \leqq K \quad \text { for } x \in\langle b-\eta, b\rangle \text {, }
$$

(where $\alpha$ and $K$ are suitably chosen positive numbers, depending only on $f(x)$ and $s$, and endowed with the metric

$$
\rho[\phi, \psi]=\sup _{\langle b-\eta, b\rangle}\left|\phi^{\prime \prime}(x)-\psi^{\prime \prime}(x)\right|,
$$

(with which $R$ becomes a complete metric space). Consider the transformation

$$
\Phi[\phi]=h(\phi[f(x)]) .
$$

This transformation (under a suitable choice of $\eta, \alpha$ and $K$ ) maps $R$

${ }^{2}$ Of course, for $s \in(-\infty, \infty)$ the values of $\left[f^{\prime}(b)\right]^{3}$ run over the set of all positive numbers. However, in order that $\phi_{s}(x)$ form a family of continuous iterations of $f(x)$ (cf. (9) below), it is convenient to define $\phi_{s}(x)$ with the aid of relation (6).

In his paper [2] B. Choczewski deals with the more general equation $\phi(x)$ $=H(x, \phi[f(x)])$. 
into itself. Furthermore, in view of (5), we have, for $\phi, \psi \in \Omega$

$$
\rho[\Phi[\phi], \Phi[\psi]]<\vartheta \rho[\phi, \psi], \quad \vartheta<1 .
$$

Consequently, $\Phi$ is a contraction mapping, and by the theorem of Banach-Caccioppoli, has exactly one fixed point in $R$, i.e. there exists exactly one function $\phi_{s}(x)$ of class $C^{2}$ in $\langle b-\eta, b\rangle$, satisfying equation (4) and conditions (7). This function can be uniquely extended onto the whole interval $(a, b)$ in such a manner that it will satisfy equation (4).

Hence it follows (see also [3, Theorem V]) that the function $\phi_{s}(x)$ can be obtained as the limit of the sequence defined by the recurrence formula

$$
\phi_{s, n+1}(x)=h\left(\phi_{s, n}[f(x)]\right), \quad n=0,1,2, \cdots,
$$

where $\phi_{s, 0}(x)$ is an arbitrary function which, when restricted to $\langle b-\eta, b\rangle$, belongs to $R$, and thus e.g. an arbitrary function of class $C^{2}$ in $(a, b\rangle$, fulfilling conditions (3). One can easily verify that formula (2) follows immediately from (8).

From the theorems of $B$. Choczewski [2] it further follows that the function $\phi_{s}(x)$ has the same regularity properties as $f(x)$.

To complete the proof of our theorem it remains only to show that the functions $\phi_{s}(x)$ form a family of continuous iterations of $f(x)$, i.e. that they satisfy the relation

$$
\phi_{s}\left[\phi_{t}(x)\right]=\phi_{s+t}(x), \quad x \in(a, b\rangle, \quad s, t \in(-\infty, \infty) .
$$

Now, the function $\phi_{s}\left[\phi_{t}(x)\right]$, just like $\phi_{8}(x)$ and $\phi_{t}(x)$, is of class $C^{2}$ in $(a, b)$ and evidently commutes with $f(x)$. We have also $\phi_{s}\left[\phi_{t}(x)\right]$ $\in(a, b\rangle \quad$ for $\quad x \in(a, b\rangle, \quad \phi_{s}\left[\phi_{t}(b)\right]=b,\left.\quad(d / d x) \phi_{s}\left[\phi_{t}(x)\right]\right|_{x=b}$ $=\phi_{s}^{\prime}\left[\phi_{t}(b)\right] \phi_{i}^{\prime}(b)=\phi_{s}^{\prime}(b) \phi_{t}^{\prime}(b)=\left[f^{\prime}(b)\right]^{s+t}$. But the function with these properties is unique. Thus $\phi_{s}\left[\phi_{t}(x)\right]=\phi_{s+t}(x)$, which was to be proved.

REMARK. The above theorem is closely related to some results of L. Berg [1]. The results obtained by Berg are, in some respect, stronger than ours - the condition $b<\infty$ does not occur, and the case $f^{\prime}(b)=1$ is also discussed. On the other hand, in most of the theorems in [1] assumptions of analyticity, or at least of multiple differentiability of $f(x)$, as well as the assumption that $f^{\prime \prime}(x)$ has a constant sign, occur; while in our case the assumptions regarding the regularity of $f(x)$ are weaker.

Similar equations have been also investigated by S. Sternberg [5]. He has obtained similar results, however in a different way.

It follows from [4] that in condition (A) it is not sufficient to re- 
quire only the continuity of $\phi_{s}(x)$ : for equation (1) admits continuous solution depending on an arbitrary function. Unfortunately, however, our method does not allow us to decide whether in addition to $\phi_{s}(x)$ there also exist other functions of class $C^{1}$ in $(a, b)$ and fulfilling conditions (B) and (C). (For the linear function; $f(x)=b+q(x=b)$ such functions do not exist; cf. $[1, \S 1]$.)

\section{REFERENCES}

1. L. Berg, Iterationen von beliebiger Ordnung, Z. Angew. Math. Mech. 40 (1960), 215-229.

2. B. Choczewski, Investigation of the existence and uniqueness of differentiable solutions of a functional equation, Ann. Polon. Math. (to appear).

3. J. Kordylewski and M. Kuczma, On the functional equation $F(x, \phi(x), \phi[f(x)])$ $=0$, Ann. Polon. Math. 7 (1959), 21-32.

4. M. Kuczma, On continuous solutions of a functional equation, Ann. Polon. Math. 8 (1960), 209-214.

5. S. Sternberg, Local $C^{n}$ transformations of the real line, Duke Math. J. 24 (1957), 97-102.

Uniwersytet Jagielloński, Kraków, Poland 